\title{
The neural underpinnings of reading skill in deaf adults
}

\author{
Karen Emmoreya \\ Stephen McCullough ${ }^{\mathrm{a}}$ \\ Jill Weisberga
}

aSan Diego State University

Laboratory for Language and Cognitive Neuroscience

6495 Alvarado Road, Suite 200

San Diego, CA 92012

USA

Corresponding author:

Karen Emmorey

San Diego State University

6495 Alvarado Road, Suite 200

San Diego, CA 92012

USA

kemmorey@mail.sdsu.edu

(619) 594-8080 


\begin{abstract}
We investigated word-level reading circuits in skilled deaf readers $(\mathrm{N}=14$; mean reading age $=19.5$ years $)$ and less skilled deaf readers $(\mathrm{N}=14$; mean reading age $=12$ years $)$ who were all highly proficient users of American Sign Language. During fMRI scanning, participants performed a semantic decision (concrete concept?), a phonological decision (two syllables?), and a false-font control task (string underlined?). No significant group differences were observed with the full participant set. However, an analysis with the 10 most and 10 least skilled readers revealed that for the semantic task (vs. control task), proficient deaf readers exhibited greater activation in left inferior frontal and middle temporal gyri than less proficient readers. No group differences were observed for the phonological task. Whole-brain correlation analyses (all participants) revealed that for the semantic task, reading ability correlated positively with neural activity in the right inferior frontal gyrus and in a region associated with the orthography-semantics interface, located anterior to the visual word form area. Reading ability did not correlate with neural activity during the phonological task. Accuracy on the semantic task correlated positively with neural activity in left anterior temporal lobe (a region linked to conceptual processing), while accuracy on the phonological task correlated positively with neural activity in left posterior inferior frontal gyrus (a region linked to syllabification processes during speech production). Finally, reading comprehension scores correlated positively with vocabulary and print exposure measures, but not with phonological awareness scores.
\end{abstract}

Keywords: deaf; word reading; semantics; phonology; fMRI 
Very little is known about the neural adaptations that support skilled reading when the process of learning to read is altered by deafness rather by a specific reading disability. Reading presents a challenge for the majority of deaf children because they do not receive the same everyday exposure as hearing children to the language that is encoded by print. Hence, most deaf children begin reading instruction without the benefit of the strong language foundation and well-established pedagogical methods available to their hearing peers. Although reading outcomes are generally poor for deaf people, some deaf individuals do, nonetheless, achieve high levels of reading proficiency (Qi \& Mitchell, 2012). However, how such skilled readers differ from less-skilled deaf readers is poorly understood because most investigations simply compare groups of deaf and hearing readers without regard to the variability of reading skill or language background within the deaf group. The goal of the present study was to identify possible neural signatures that differentiate between skilled and less-skilled reading in deaf adults.

This study focuses on prelingually deaf adults with severe-to-profound hearing loss who acquired American Sign Language (ASL) in early childhood. We target this population because they form a relatively homogeneous group and they are less likely to access phonological codes when reading, compared to "oral" deaf readers who acquire only a spoken language (e.g., Koo, Kelly, LaSasso, \& Eden, 2008; Hirshorn, Dye, Hauser, Supalla, \& Bavelier, 2015). One question of interest is whether and how impoverished (e.g., less precise) phonological representations of speech affect the reading system in deaf readers. Emerging evidence suggests that phonological abilities may be only weakly related to reading skill for deaf people (Mayberry et al. 2010; Miller \& Clark, 2011), although this idea is controversial (Paul et al. 2009). Here we used fMRI to examine both phonological and 
semantic processing of English words by deaf readers whose reading levels ranged from the fourth grade ( 9 years) to college level (22 years). One aim of the study was to determine whether the less-skilled readers rely on a distinct neural circuit when reading (for meaning or when extracting speech-based phonological information) compared to skilled deaf readers.

In a recent fMRI study of implicit word reading (detect an ascending letter/symbol in words vs. false-font strings), Corina, Lawyer, Hauser, and Hirshorn (2013) reported that relatively skilled deaf readers (mean reading age $=14$ years) engaged several brain regions (left superior temporal gyrus (STG), bilateral fusiform gyri, and bilateral inferior parietal lobules) to a greater extent than less skilled deaf readers (mean reading age $=9.9$ years). Separate examination of the less-skilled readers $(p<.005$, uncorrected) revealed bilateral activation in the middle frontal gyrus (BA 46/9), which was not observed for the more skilled readers. Based on these activation patterns, Corina et al. (2013) suggested that less skilled deaf readers may "process English word forms as non-decomposable logographiclike forms analogous to Chinese (pg. 6)." However, the between-group contrast did not reveal greater activation in the middle frontal gyri (or in any brain area) for the less-skilled deaf readers, even at uncorrected thresholds, and thus this characterization must be considered tentative.

Using a lexical decision task (and a feature detection task with unreadable symbols as the baseline), Aparicio, Gounot, Demont, and Metz-Lutz (2007) found that less skilled deaf readers (all bilingual in French and French Sign Language) exhibited greater activation in the right inferior frontal gyrus (IFG) and left STG, as well as reduced activation in left IFG compared to more skilled hearing readers. However, these differences were only evident in 
region of interest (ROI) analyses - not in the whole-brain analysis. Li, Peng, Lui, Booth, and Ding (2014) found a similar (and statistically more robust) pattern for deaf Chinese readers making semantic relatedness judgments to pairs of Chinese characters. The deaf readers (fluent users of Chinese Sign Language) showed greater activation in right frontal cortex (as well as in the right inferior parietal lobule) and reduced activation in left IFG compared to hearing readers (reading ability was not assessed). Li et al. (2014) suggested that greater recruitment of right hemisphere regions reflect a reliance on sign language processing during reading because sign language engages right frontal and parietal regions to a greater extent than text processing (e.g., Neville et al., 1998). The two groups of deaf participants in the current study differed in reading ability but were equally proficient in ASL, allowing us to determine whether recruitment of the right hemisphere reflects a compensatory mechanism related signed language use.

In a previous fMRI study, Emmorey, Weisberg, McCullough, and Petrich (2013) compared the same highly skilled deaf readers from the current study with hearing peers who were matched for reading ability. Participants made semantic decisions (concrete/abstract?) or phonological decisions (two syllables?) to English words, and during a control task, made decisions to false font strings (underlined?). For the semantic processing task, Emmorey et al. (2013) reported strongly left-lateralized neural activity for both groups and found no regions where activation significantly differed between skilled deaf and hearing readers. These skilled deaf readers did not differentially recruit right hemisphere regions nor did they exhibit distinct activation patterns within the left hemisphere when reading words for meaning - the deaf and hearing readers both engaged left IFG, left superior temporal sulcus, and left inferior temporal gyrus (including the 
putative Visual Word Form Area, VWFA; Cohen et al., 2002). In the study reported here, we compared the neural responses of these skilled deaf readers with a group of less-skilled deaf readers performing the same tasks to investigate how reading skill modulates activation within the reading circuit for deaf adults who are fluent in a sign language.

Although Emmorey et al. (2013) found no differences between skilled deaf and hearing readers for semantic decisions, they observed robust differences in neural activity when participants judged the number of syllables within a word. This phonological task was significantly more difficult for the deaf readers, and they exhibited increased activation (relative to hearing readers) in the intraparietal sulcus bilaterally and in the left precentral gyrus. Emmorey et al. (2013) suggested that these findings reflect greater processing demands for deaf, compared to hearing individuals, when making speech-based phonological decisions (parietal increases), and greater reliance on articulatory representations of speech for deaf compared to hearing people (left frontal increase) (see also MacSweeney, Brammer, Waters, \& Goswami, 2009). Other studies have also found greater recruitment of inferior parietal cortex for deaf compared to hearing readers when performing phonological tasks, e.g., rhyme judgments (Aparicio et al., 2007; Li et al., 2014). In the present study, we investigated whether neural activity differs for less skilled versus skilled deaf readers when performing the syllable judgment task. Given that a metaanalysis by Mayberry et al. (2010) indicated that phonological ability was not strongly predictive of reading level for deaf individuals, we may find that reading skill does not modulate neural activity during phonological processing tasks for deaf readers.

In addition, we take advantage of the variability in reading skill across this large group of deaf participants $(\mathrm{N}=28)$ to examine brain-behavior correlations between neural 
activity and reading ability, as well as between neural activity and performance on the semantic and phonological tasks. One question of interest is whether activation within the VWFA increases with reading skill for deaf readers, as has been found for hearing readers (McCandliss, Cohen, \& Dehaene, 2003). Corina et al. (2013) reported greater activation in the mid fusiform gyrus bilaterally (within the range of locations reported for the VWFA) for skilled relative to less skilled deaf readers. In contrast, Wang et al. (2014) found that the anatomical location and activation strength of the VWFA were highly similar between deaf and hearing Chinese readers despite the fact that the deaf readers (all signers) had lower reading levels than the hearing readers. Similarly, Aparicio et al. (2007) and Waters et al. (2007) reported no activation differences in this region when deaf readers were compared to hearing readers with better reading ability.

The brain-behavior correlation analyses for task performance also provide clues as to the linguistic or cognitive operations that support word level semantic and phonological processing for deaf readers. For example, a positive correlation between syllable judgment accuracy and neural activity in left prefrontal cortex will suggest that success at phonological tasks is associated with greater use of articulatory codes (see MacSweeney et al., 2009), while a correlation with parietal activity will suggest that success is associated with mapping orthographic to phonological codes (see Pugh et al., 2000).

\section{Method}

\section{Participants}

Twenty-eight deaf ASL signers participated in the study; half were skilled readers (reading level at or above $10^{\text {th }}$ grade, or age 15 years) and half were less-skilled readers (reading level at or below $9^{\text {th }}$ grade, or age 14 years), based on the reading comprehension 
subtest of the Peabody Individual Achievement Test (PIAT). This test requires participants to read a sentence and decide which of four pictures best matches that sentence. As the test progresses, the sentences become longer, contain a greater number of clauses, and employ less frequent vocabulary. As can be seen in Table 1, on average the skilled group had a reading age equivalent of 19.5 years (college level) while the less-skilled group had a mean reading age equivalent of 12 years (middle school grade level). The skilled readers had significantly higher vocabulary scores based on the Shipley Vocabulary test (Shipley, 1940) and greater print exposure based on the Author and Magazine Recognition Tests (Stanovich and West, 1989). The skilled readers also scored higher on the nonverbal component of the Kaufman Brief Intelligence Test (Kaufman \& Kaufman, 2004) than the less-skilled readers. We therefore used this measure of non-verbal IQ (NVIQ) as a covariate for between-group MRI analyses and the correlation analyses.

The reading groups did not differ on a phonological awareness test $(t<1)$, which required participants to select two pictures (out of four) that began or ended with the same sound (adapted from Miller, 1997). Prior to testing, participants were asked to provide the English label for each picture (separately), and if they provided a different label, the experimenter indicated the correct target English word. An example of an initial sound trial is the following: knee, key, fork, nurse; an example of a final sound trial is: book, gum, comb, crab. For all trials, the task could not be solved based on orthography alone. Performance variability was high, and both groups scored relatively poorly on this test (52\% and $45 \%$ correct) compared to hearing readers, e.g., hearing participants scored $88 \%$ correct on this test in the Emmorey et al. (2013) study. Nonetheless, both deaf groups clearly performed above chance (16\%). 
Participants were also asked to indicate on a 5-point scale how frequently they used speech to communicate with hearing people (where $1=$ "not at all" and $5=$ "very much") and how much they understood when lip-reading a hearing person $(1=$ "not at all"; $5=$ "very much"). Both reading groups indicated relatively low use of speech (mean rating for skilled $=2.4$, less-skilled $=2.0)$ and moderate lip-reading ability (mean rating for skilled $=$ 2.9 , less-skilled $=3.0$ ), indicating that neither group relied heavily on spoken language in their everyday lives. The finding that speech abilities did not differentiate skilled from lessskilled deaf readers replicates the findings of Chamberlain and Mayberry (2008), who were the first to report that reading experience (e.g., print exposure, vocabulary knowledge), rather than self-reported speech use or comprehension, was associated with better reading ability for deaf signing adults.

The skilled and less-skilled reading groups were balanced for gender and age (see Table 1). The groups were also balanced for ASL experience, with ten native signers (born into deaf signing families) in the skilled group and eleven native signers in the less-skilled group. The remaining signers in each group all learned ASL prior to age five. Participants' ASL ability was assessed with the long version (35 sentences) of the ASL Sentence Reproduction Test (ASL-SRT; Supalla, Hauser, and Bavelier, 2014). ASL-SRT scores did not differ between the skilled and less-skilled readers.

All participants were right-handed and severely-to-profoundly deaf $(70 \mathrm{~dB}$ hearing loss or greater), had 12 or more years of formal education, reported no neurological or behavioral disorder, and no history of learning disability. Participants were recruited from the southern California metropolitan areas of Los Angeles and San Diego via flyers and 
word-of-mouth referrals. Informed consent was obtained according to procedures approved by the UCSD and SDSU Human Research Protection Programs.

\section{Materials}

The stimuli for the semantic decision task were designed as part of a larger study in which the same items were also presented as either fingerspelled words or ASL signs (see Emmorey et al., 2015). Here we report only results from printed word stimuli, comparing skilled and less-skilled deaf readers (see Emmorey et al., 2013, for a comparison of the skilled deaf readers with matched hearing readers). To counterbalance stimuli presentation, we created two lists of 80 words (half concrete, half abstract) that were matched for frequency, length, and concreteness. Half of the words had ASL sign translations and half did not. For the phonological decision task we created a separate list of 80 words that did not appear in the semantic word set, but were matched for frequency, word length, and concreteness with the words in the semantic set. Half of the words in the phonological set contained two syllables and the other half did not (approximately equal numbers of words with one syllable or more than two syllables). We ensured that access to phonology was required to successfully perform the task by controlling the orthographic length of stimuli such that length did not predict the number of syllables (e.g., lion vs. cake; greedy vs. denial; trolley vs. caravan). The stimuli for the control task consisted of 80 false font strings (unintelligible symbols designed to resemble letters of a foreign script) borrowed from Price, Wise, and Frackowiak (1996). Each false font character was yoked to a specific letter of the alphabet, and the false font "words" were matched for length with the English words from the experimental conditions. Half of the false font strings were underlined and half were not. See Emmorey et al. (2013) for further details regarding 
stimulus materials.

\section{fMRI Design and Procedure}

The fMRI paradigm consisted of 30-second experimental blocks (semantic decision, phonological decision) and 30-second control (false font) blocks presented in pseudorandom order. There were two runs (8 min each) of four complete stimulus cycles. Each cycle included a phonological task block, a semantic task block, a false font task block, and a 16s fixation period (at the end of each cycle). A 3s cue printed in green ("Concrete?"; “2 syllables?"; "Underlined?") preceded each block (except fixation), instructing participants to perform the target task. Each stimulus was presented in black 48-point Arial Bold for $1000 \mathrm{~ms}$, with a $2000 \mathrm{~ms}$ interstimulus interval that contained a task reminder cue (C?; 2?; U?), printed in grey on a white background (for further details see Emmorey et al., 2013).

The stimuli were projected onto a screen at the foot of the scanner bed, using an XGA video projector and MacBook Pro computer running PsyScope X Build 53 (psy.ck.sissa.it). Participants viewed the screen through a mirror placed atop the head coil, and responded using an MR-safe Lumitouch 4-button response box (Photon Control, Burnaby, Canada), pressing one button for 'yes' responses and another for 'no' responses. For the semantic task, participants decided whether each word represented a concrete entity or not; "concrete" was defined as anything that could be touched. For the phonological task, participants decided whether each word had two syllables or not. For the control task, participants saw a string of "false font" symbols and decided whether the string was underlined or not. Response hand was counterbalanced across participants. For all tasks, participants were encouraged to respond as quickly and accurately as possible 
and were told that if a word was unfamiliar to them, they could either skip that item (i.e., not respond) or make their best guess. Instructions were presented in ASL and in written English. Participants performed three blocks of practice trials for each task outside the scanner (with items not seen during scanning).

\section{Data acquisition}

MRI data were collected at the Center for Functional MRI at the University of California, San Diego, on a 3-Tesla GE MR750 scanner equipped with an eight-channel head coil. For anatomical reference and spatial normalization, we acquired high-resolution structural images of each participant's brain at the beginning of the session using a T1weighted Fast Spoiled Gradient-Recalled Echo sequence (FOV 256mm, 256 x 256 matrix, 1 $\mathrm{mm} \times 1 \mathrm{~mm}$ in-plane resolution, $1761 \mathrm{~mm}$ thick sagittal slices, flip angle $=8^{\circ}$, inversion time $=600 \mathrm{~ms}$ ). For functional images, we acquired $40 \mathrm{~T} 2 *$-weighted, gradient-echo echoplanar (EPI) axial slices interleaved from inferior to superior, covering the whole brain, with a repetition time (TR) of $2000 \mathrm{~ms}$, an echo time (TE) of $30 \mathrm{~ms}$, flip angle $=90^{\circ}, \mathrm{FOV}=$ $224 \mathrm{~mm}, 64$ x 64 matrix, 3.5 x $3.5 \mathrm{~mm}$ in-plane resolution, and $3.5 \mathrm{~mm}$ slice thickness (no gap). During the functional scans, we first collected two printed word runs of 240 EPI volumes from each participant, and in the same session, two runs of 270 EPI volumes each for fingerspelled words and ASL signs (results reported in Emmorey et al., 2015). Four additional volumes were added to the beginning of each functional run to allow magnetization to reach steady state before stimulus presentation. These "dummy" volumes were removed during data pre-processing. 


\section{Data preprocessing and analysis}

All MRI data were preprocessed and analyzed using the AFNI software package (version AFNI_2010_10_19_1028; Cox, 1996). Anatomical and EPI volumes for each participant were spatially normalized to standardized space (Talairach \& Tournoux, 1988) using the AFNI TT_N27 template (Colin27) and @auto_tlrc program, which performs a 12parameter affine registration. After discarding the first four time points, EPI volumes were corrected for slice timing (3dTshift) and head movements (3dvolreg using Fourier interpolation and the second volume of the first run as the reference). Estimates of the three translation and three rotation parameters were examined with 1d_tool.py to identify any unusual spikes in the functional data and were saved for use during statistical analysis. Any time points where the square root of the sum of squares (Euclidean norm) of the derivatives exceeded one were censored from statistical analysis. The functional data were smoothed with a $7 \mathrm{~mm}$ Gaussian filter and scaled to a mean of 100 by dividing each voxel's value by its mean across time points to derive percent signal change.

Each individual's printed word runs were analyzed using AFNI's multiple regression program (3dREMLfit). Three regressors of interest were modeled using AFNI's BLOCK basis function with a duration of 30 seconds: one regressor each for the semantic, phonological, and false font decision blocks. Nuisance variables in the regression model included the six motion parameters obtained during motion correction and a Legendre polynomial set ranging from zero to third order for each run to account for slow drifts. Fixation periods comprised the baseline for the regression model and thus were not explicitly modeled with a regressor. Beta weights were calculated for each stimulus type and for the contrast of each experimental task (semantic decision, phonological decision) 
versus the false font control task. To examine whole-brain within-group level effects, individuals' voxel-wise response estimates (beta-weights) and their corresponding $t$-values for each stimulus type and/or contrast of interest served as inputs to mixed effects meta analyses (3dMEMA; Chen, Saad, Nath, Beauchamp, \& Cox, 2012). We conducted betweengroup mixed effects meta analyses separately for the semantic and phonological tasks using individuals' responses estimates and corresponding t-scores from the relevant contrast (i.e. semantic task vs. false font control task; phonological task vs. false font control task). These between-group analyses (i.e., skilled readers (experimental task - control task) vs. less skilled readers (experimental task - control task) included z-transformed NVIQ scores as a covariate and accounted for unequal variance across groups. Unless otherwise noted, we used a false discovery rate (FDR) correction for multiple comparisons (Benjamini \& Hochberg, 1995), and report all clusters of 10 or more contiguous voxels exceeding an FDR corrected threshold of $q<.05$. For all figure illustrations, MRI statistical maps were resampled to $1 \mathrm{~mm}^{3}$ resolution using cubic interpolation.

We used the same approach for a post-hoc analysis comparing subgroups comprised of the 10 most and 10 least skilled readers. In light of the reduced power afforded by the smaller sample size in this analysis, we report activations that surpassed a slightly less conservative significance threshold of $p<.01$, implemented in combination with a minimum cluster size criterion of 15 contiguous voxels $\left(643 \mathrm{~mm}^{3}\right)$. This joint intensity-spatial extent correction for multiple comparisons balances protection against Type I errors with avoidance of Type II errors, thus limiting both the risk of false alarms and the risk of treating real effects as spurious, i.e. increasing the likelihood of detecting subtle, but true effects (see Lieberman \& Cunningham, 2009). 
Lastly, we conducted correlation analyses across all 28 participants to examine the relationship between behavioral performance and brain responses (see Results for details).

\section{Results}

\section{Behavioral results}

Response times (RTs) and accuracy (percent correct) for the experimental tasks are listed in Table 1. RTs did not differ significantly between the skilled and less-skilled readers for the semantic task, $t(27)=.81, p=.42$, the phonological task, $t(27)=1.52, p=.14$, or the false-font control task (skilled readers $=751 \mathrm{~ms} ; S D=242 \mathrm{~ms}$ and less-skilled readers $=$ $726 \mathrm{~ms} ; S D=85 \mathrm{~ms}), t(27)<1, p=.72$. However, the skilled readers were significantly more accurate than less-skilled readers for both the semantic task, $t(27)=4.06, p<.001$ and the phonological task, $t(27)=3.03, p<.01$. Accuracy for the control task did not differ between groups (skilled readers $=99.46 \% ; S D=0.64 \%$ and less-skilled readers $=99.11 \%$; $S D=1.14 \%), t(27)=1.01, p=.32)$.

We also examined the relationship between our reading and phonological assessment measures, and between those measures and performance on the in-scanner experimental tasks (with variance due to NVIQ partialled out). The partial correlation results are given in Table 2. Reading level (PIAT scores), print exposure (Author and Magazine Recognition tests), and vocabulary (Shipley) were all highly correlated with each other. In contrast, phonological awareness was not correlated with any of these reading measures. Accuracy on the in-scanner phonological task (syllable judgment) correlated with phonological awareness, vocabulary, and PIAT scores. Accuracy on the in-scanner semantic task (concreteness judgment) correlated with all of the reading measures, but not with performance on the phonological awareness test. 
Note that the high correlation between in-scanner behavioral accuracy (on the semantic and phonological tasks) and our grouping variable (PIAT scores) indicate that they likely share variance. Thus, it is inappropriate to include behavioral performance as a covariate for out fMRI analyses (Chapman \& Miller, 2001). Partialling out variance associated with in-scanner task performance would also partial out real group differences in brain activation related to reading skill (our independent variable).

\section{Neuroimaging results}

Semantic processing. In both skilled and less-skilled deaf readers, the semantic decision task yielded greater activation than the control task in frontotemporal language regions, with predominantly left hemisphere activation in both groups (including left inferior frontal gyrus (BA 45) and middle temporal gyrus; see Table 3). The direct contrast between groups revealed no regions significantly more active for one group than the other, possibly due to more heterogeneity in the location and strength of activation for the lessskilled readers. Inspection of variance across subjects (voxel-wise tau ${ }^{2}$ values generated by 3dMEMA) revealed a high degree of heterogeneity in the location of activation across the less skilled readers.

In addition, there may not have been a sufficient difference in reading level between the two groups to detect a significant difference in BOLD response. Therefore, we conducted a follow-up analysis comparing the 10 most skilled readers with the 10 least skilled readers based on PIAT scores (see Table 1 for the behavioral and demographic characteristics of these subgroups). As shown in Figure 1, this contrast revealed significantly greater activation for skilled readers in two regions: left inferior frontal gyrus 
(peak in BA 44/6; $-57,+10,+17 ; \mathrm{vol}=943 \mathrm{~mm}^{3} ; t=5.1$ ) and left middle temporal gyrus (peak in BA 21/37; $-47,-43,+3 ; \mathrm{vol}=1009 \mathrm{~mm}^{3} ; t=4.02$ ). No regions were more active for the less skilled than the skilled readers in this subgroup analysis.

Phonological processing. The full groups of skilled and less-skilled deaf readers both exhibited greater activation for the phonological decision compared to the control task in bilateral frontal cortices (with activation extending into the insula) and in bilateral inferior parietal cortex, with peaks in the intraparietal sulcus (Table 4). In addition, we observed increased activation in left inferior temporal cortex in both groups (peaks for skilled and less skilled readers in the fusiform gyrus and parahippocampal gyrus, respectively). As with the semantic task, the direct contrast revealed no regions of differential activation between groups. In contrast to the semantic task, the subset analysis with the top and bottom 10 readers revealed no significant group differences for the phonological task.

\section{Brain-behavior correlation analyses}

For the whole-brain correlation analyses, we combined the skilled and less skilled deaf readers into a single large group $(N=28)$. Using AFNI's 3dRegAna multiple linear regression program, we examined whether brain responses during the semantic or phonological tasks (vs. the false font control task) correlated with reading ability or with the task accuracy data collected during scanning. Rather than separately assessing each of the four highly correlated reading scores in this analysis (i.e. the PIAT, ART, MRT, and Shipley Vocabulary Test), we used a single composite score of reading ability for each participant. We computed the composite reading score following the procedures outlined in Schneider (2013), using the following calculation, where $k=$ number of tests; $\square_{\square}=$ test index score; $\square_{\square \square}=$ correlation coefficient for each row $i$ and column $j$ : 
Composite Score $=\frac{\sum_{i=1}^{k}\left(X_{i}\right)-100 k}{\sqrt{\sum_{i=1}^{k} \sum_{j=1}^{k}\left(r_{i j}\right)}}+100$

Each participant's composite reading score was z-transformed prior to correlation analyses. As with the between-group contrasts, we included z-transformed NVIQ scores (K-BIT scores) as a covariate.

The partial correlation statistical maps were corrected for multiple comparisons to $p<.05$, using a combination of alpha and minimum cluster size determined by Monte Carlo simulations (AFNI's 3dClustSim). Individual values in the surviving group-level clusters were plotted and inspected for large outliers that might skew the results, i.e., greater than 3 sigma (using the R statistical package; R Core Team, 2011), and none were found.

Semantic processing. As shown in Figure 2A, for the semantic task, there was a significant positive partial correlation $\left(\alpha<.01\right.$, minimum cluster volume $\left.=1329 \mathrm{~mm}^{3}\right)$ between neural activity and task accuracy in a cluster of voxels located in left anterior temporal lobe (Volume $=2572 \mathrm{~mm}^{3} ;-47,-5,-29$; peak $t=+5.47$ ). Partial correlation coefficients across voxels within this cluster ranged from $r=.44-.73$. Thus, higher accuracy on the concreteness decision task was associated with stronger BOLD responses in this region. In addition, two regions showed a significant positive correlation between reading ability and neural activity during semantic processing $(\alpha<.005$, minimum cluster volume $=900 \mathrm{~mm}^{3}$ ): one region was focused in the left inferior temporal gyrus, anterior to the visual word form area (Volume $=943 \mathrm{~mm}^{3} ;-54,-29,-21$; peak $t=+5.02 ; r$ range $=.53$ -.71 ), and the other was focused in the right inferior frontal gyrus (Volume $=1972 \mathrm{~mm}^{3}$; $+47,+34,+3$; peak $t=+4.46 ; r$ range $=.54-.67)$.

Phonological processing. Neural activity during the phonological task correlated 
positively with task accuracy $\left(\alpha<.01\right.$, minimum cluster volume $\left.=1329 \mathrm{~mm}^{3}\right)$ in two clusters located in the left inferior frontal gyrus: in BA 44 (Volume $=1586 \mathrm{~mm}^{3} ;-58,+10$, +17 ; peak $t=+6.47 ; r$ range $=.49-.79)$ and in BA 44/45 (Volume $=1843 \mathrm{~mm}^{3} ;-33,+24$, +21 ; peak $t=+3.49 ; r$ range $=.49-.57$ ) (Figure 2B). In these clusters, higher accuracy on the syllable decision task was associated with increased neural activity. We observed no significant correlations between neural activity and reading ability for the phonological task.

\section{Discussion}

Skilled and less-skilled deaf readers showed similar patterns of neural activation for both the semantic and the phonological processing tasks, although the less skilled group exhibited less extensive activation within the reading circuit (see Tables 3 and 4). The full group analyses revealed no significant between-group differences in neural activation for either task, perhaps because the groups were not distinct enough in reading ability. A follow-up analysis with the ends of our reading score distribution revealed significant group differences in activation for the semantic task (concreteness judgment), but not for the phonological task (syllable number judgment). The lack of significant differences in neural activity between the reading subgroups for the phonological task could be due, in part, to the wide variability in performance on that task (particularly for the skilled readers; see Table 1).

When reading words for meaning, the most skilled deaf readers in our study exhibited greater activity in the left inferior frontal gyrus and left middle temporal gyrus, compared to the least skilled readers. Interestingly, this pattern contrasts with the results 
of Welcome and Joanisse (2012) for hearing adult readers who varied in reading ability. In that study, participants made semantic category judgments to pairs of words, and greater activation in frontal and temporal regions was associated with less skilled reading. Thus, the neural pattern associated with poorer reading ability may be different for hearing and deaf adults within the general population (i.e., non-dyslexic readers). For hearing people, decreased activation in left frontal and temporal regions may be associated with better reading because skilled hearing readers may rely less on holistic lexical/semantic processing of words compared to less skilled readers. In contrast, for deaf people, increased activation in these regions may be associated with better reading because it is the skilled deaf readers who rely more on lexical processing of words (e.g., via strong orthographicsemantic mappings) compared to less skilled deaf readers.

We found little evidence that less skilled deaf readers were more likely than skilled readers to engage right hemisphere regions when reading single words for meaning or when extracting speech-based phonological information. In fact, activation in the right inferior frontal gyrus for the semantic task was positively correlated with reading ability. However, given the role of right IFG in attention and control (e.g., Hampshire et al., 2010; Aron, Robbins, \& Poldrack, 2004), the correlation with reading ability could indicate that better deaf readers are more likely to engage cognitive control mechanisms when performing semantic decisions about words (e.g., selecting and attending to semantic features that are relevant to the concreteness decision). Since all of the deaf readers were skilled ASL signers, we suggest this correlation is not specifically related to use of a signed language.

Consistent with previous studies comparing deaf readers with more skilled hearing 
readers (Wang et al., 2014; Aparicio et al., 2007; Waters et al., 2007), we found no difference in neural activity in the visual word form area (VWFA) related to reading skill. This suggests that a primary marker of disabled reading in hearing individuals, namely reduced activation compared to skilled readers in the VWFA (e.g., Hoeft et al., 2007), does not hold for less skilled deaf adult readers. However, it is possible that reading skill-related differences in VWFA activity might be observed for sentence level reading tasks in deaf readers. Hirshorn, Dye, Hauser, Supalla, and Bavelier (2014) found weaker activation in the VWFA during sentence reading (vs. "false font" sentences) for deaf native signers compared to 'oral' deaf (non-signers) and hearing readers. However, all groups in that study had similar reading levels. In addition, Emmorey et al. (2013) found no difference in VWFA activation when reading-matched deaf signers and hearing speakers read single words. Thus, reduced VWFA activity for the deaf signers in the Hirshorn et al. study may be specific to reading sentences and appears to be unrelated to reading skill.

One could speculate that our fMRI results may be associated with task difficulty, rather than reading skill, because the skilled and less-skilled readers were not equally accurate on the tasks performed during scanning. However, this is an unlikely explanation for several reasons. We would expect group differences in accuracy on the in-scanner tasks to elicit group differences in brain activation. However, we found no group differences in activation for either task when comparing 14 skilled with 14 less-skilled readers. Second, direct comparison of fMRI responses between the 10 most and 10 least skilled readers revealed between-group differences only for the semantic task, despite significant group differences in accuracy for both the semantic and the phonological tasks. Third, the brain regions activated more for skilled than less-skilled readers during the semantic task were 
left hemisphere language regions, rather than regions associated with attention and task difficulty.

The results of our brain-behavior correlation analyses revealed that better reading ability was associated with increased neural activity within left inferior temporal cortex, in a region anterior to the VWFA, when deaf readers performed the semantic task. We hypothesize that this more anterior region is involved in mapping orthographic word-level representations onto lexical-semantic representations. Using a lesion mapping procedure with patients who had acquired dysgraphia and dyslexia, Purcell, Shea, and Rapp (2014) identified a similar region anterior to the VWFA as an important interface between orthography and semantics for both reading and spelling. Our correlation result suggests that better deaf readers may have stronger or more finely-tuned links between orthographic and semantic word-level representations, such that good readers engage this interface more consistently, or to a greater extent, than poor deaf readers processing written words for meaning.

Accuracy on the semantic decision task was correlated with neural activity in the left anterior temporal lobe (ATL). In hearing people, both neuroimaging and patient data indicate that the ATLs are involved in representing and processing semantic knowledge, with the left ATL often more involved than the right for linguistic stimuli (e.g., Gesierich et al., 2012; Lambon Ralph, Sage, Jones, \& Mayberry, 2010; Patterson, Nestor, \& Rogers, 2007; Wong \& Gallati, 2012; see also Emmorey, McCullough, Mehta, Ponto, \& Grabowski, 2013, for evidence from ASL). The fact that we observed greater involvement of left ATL with increasing task accuracy suggests that activation here may reflect depth of semantic processing when making concreteness judgments. This result also suggests that deaf and 
hearing readers rely on a similar neural substrate to support conceptual processing when reading words.

In contrast to results for the semantic task, we found no significant correlations between neural activity and reading ability for the syllable judgment task. Behaviorally, however, reading ability was significantly correlated with the accuracy of syllable judgments during scanning $(r=.55, p<.005)$, but not with the phonological awareness test. This pattern suggests that skilled deaf readers are better able to access higher-level syllabic information about words, but the ability to access sub-syllabic information about initial or final consonants (as assessed by our phonological awareness test) is not related to reading ability. Consistent with this hypothesis, Sterne and Goswami (2000) found that compared to reading-age matched hearing controls, deaf children (mean reading age $=7.2$ years; mean chronological age $=11.8$ years) exhibited equal syllable awareness, but much poorer rhyme awareness. Rhyme judgments were more difficult and required access to subsyllabic segments. Sterne and Goswami (2000) concluded that "phonological skills [tapped by rhyme judgments] may not be crucial for the attainment of the reading levels that our deaf children had achieved (pg. 621)."

Although reading ability showed no correlation with neural activity for the syllable judgment task, accuracy on this task was significantly correlated with neural activity in the left inferior frontal gyrus (Figure 2B). The more posterior cluster we identified in the IFG (peak in BA 44) is very similar to the region that MacSweeney et al. (2009) reported as more active for deaf, compared to hearing adults, performing English rhyme judgments with picture stimuli. Following MacSweeney et al. (2009), we hypothesize that deaf readers rely more on fine-grained articulatory coding (covert pronunciation) than on auditory 
processes when reading. Further, syllabification processes during speech production have been linked to activation in left posterior IFG (e.g., Indefry \& Levelt, 2004). We suggest that for deaf readers, increased neural activity in this region is associated with engagement of word syllabification processes. We did not find a correlation within the left precentral gyrus (BA 6), indicating that task accuracy was not associated with use of covert articulation per se, but rather with access to and encoding of abstract syllabic structure. Emmorey et al. (2013) found that skilled deaf readers engaged the left precentral gyrus more than matched hearing readers when performing this same phonological task. Since we observed no difference between good and poor deaf readers in left precentral gyrus, it is possible that all deaf readers utilize covert articulation (or access articulatory representations) when performing phonological judgments. However, success on syllabification judgments appears to be linked specifically to activation within left posterior IFG. Finally, we found no correlations within inferior parietal cortex, suggesting that deaf readers' success on this task does not depend on regions that hearing readers engage to map orthographic to phonological representations (e.g., Pugh et al., 2000; Landi et al., 2013).

Finally, we observed strong positive correlations between reading comprehension (assessed by the PIAT) and measures of print exposure (the Author and Magazine Recognition tests) and vocabulary knowledge (see Table 2), but none of these measures correlated with a measure of phonological awareness. Chamberlain and Mayberry (2008) also found a strong positive correlation between print exposure and reading comprehension for adult deaf ASL signers (and no correlations with self-reported speech ability). Although increased reading experience is likely to be beneficial, correlation data 
cannot determine the direction of these relationships. For example, those who struggle with reading are likely to read less, while those who are good readers are more likely to enjoy reading and thus read more (Stanovich, 1986; Marschark et al., 2012).

In sum, we are beginning to understand the neurocognitive processes adopted by deaf readers to read printed words. In general, deaf readers engage the same reading circuit that has been identified for hearing readers (i.e., left temporal, inferior frontal, and inferior parietal cortices) when accessing semantic or phonological information from print. A post-hoc analysis with the 10 most and 10 least skilled deaf readers in our study revealed increased activation within the left inferior frontal gyrus and left middle temporal cortex for better readers when reading words for meaning. We hypothesize that increased activation in these regions reflects increased lexical-semantic processing of words by the skilled deaf readers. The brain-behavior correlation analyses revealed that during the semantic task, higher reading ability was associated with increased activation in a region anterior to the VWFA that has been associated with mapping orthographic to semantic lexical representations. Better reading skill was also associated with increased activation in right IFG, possibly indicating that stronger reading skills are linked to increased use of control processes or to greater semantic processing when reading print. Consistent with this idea, deaf readers who made more accurate concreteness judgments showed stronger neural activity in left ATL, a region associated with conceptual semantic processing. For the syllable judgment task, higher accuracy was associated with increased activation in left posterior IFG (BA 44), a region associated with syllabification processes during speech production, but we found no correlation between task accuracy and neural activation in parietal cortex. Overall, the results from this work suggest that skilled word reading in deaf 
individuals is associated with robust engagement of the same reading circuit as in hearing readers, and specifically with recruitment of regions associated with lexical-semantic processing and the orthographic-semantic interface.

\section{Acknowledgements}

This research was supported by National Science Foundation (NSF) Grants awarded to Karen Emmorey and San Diego State University (BCS-0823576; BCS-1154313) and by NSF Grant SBE-0541953 awarded to Gallaudet University for the Visual Language and Visual Learning (VL2) Science of Learning Center. We would like to thank Allison Bassett, Lucinda O'Grady Farnady, and particularly Jennifer Petrich for assistance with the study. We also thank all of the study participants, without whom this research would not be possible. 


\section{References}

Aparicio, M., Gounot, D., Demont, E., \& Metz-Lutz, M.N. (2007). Phonological processing in relation to reading: An fMRI study in deaf readers. NeuroImage, 35, 1303-1316.

Aron, A. R., Robbins, T. W., \& Poldrack, R. A. (2004). Inhibition and the right inferior frontal cortex. Trends in Cognitive Science, 8(4), 170-177.

Benjamini, Y. \& Hochberg, Y. (1995). Controlling the false discovery rate: A practical and powerful approach to multiple testing. J. R. Statist. Soc. B, 27, 289-300.

Chen, G., Saad, Z.S., Nath, A.R., Beauchamp, M.S. \& Cox, R.W. (2012). FMRI group analysis combining effect estimates and their variances. NeuroImage, 66, 249-260.

Cohen, L., Lehericy, S., Chochon, F., Lemer C, Rivaud S, et al. (2002) Language specific tuning of visual cortex? Functional properties of the Visual Word Form Area. Brain, 125,1054-1069.

Corina, D. Lawyer, L.A., Hauser, P. \& Hirshorn, E. (2013). Lexical processing in deaf readers: an FMRI investigation of reading proficiency. PLoS One, 8, e54696.

Cox, R. W. (1996). AFNI: software for analysis and visualization of functional magnetic resonance neuroimages. Comput Biomed Res., 29, 162-73.

Emmorey, K., McCullough, S., \& Weisberg, J. (2015). Neural correlates of fingerspelling, text, and sign processing in deaf ASL-English bilinguals. Lang Cogn Neurosci, 30, 749-767.

Emmorey, K., McCullough, S., Mehta, S.H., Ponto, L.B., \& Grabowski, T.J. (2013). The biology of linguistic expression impacts neural correlates for spatial language. J Cognitive Neurosci, 25, 517-533. 
Emmorey, K., Weisberg, J., McCullough, S. \& Petrich, J.A.F. (2013). Mapping the reading circuitry for skilled deaf readers: An fMRI study of semantic and phonological processing. Brain Lang, 126, 169-180.

Gesierich, B., Jovicich, J., Riello, M., Adriani, M., Monti, A., Brentari, V., et al. (2012). Distinct neural substrates for semantic knowledge and naming in the temporoparietal network. Cereb Cortex, 22, 2217-26.

Hampshire, A., Chamberlain, S.R., Monti, M.M., Duncan, J., \& Owen, A. M. (2010). The role of the right inferior frontal gyrus: inhibition and attentional control. NeuroImage, 50, 1313-1319.

Hirshorn, E., Dye, M. W. G., Hauser, P. C., Supalla, T., \& Bavelier, D. (2014). Neural networks mediating sentence reading in the deaf. Front in Hum Neurosc, 8, 394.

Hirshorn, E., Dye, M. W. G., Hauser, P. C., Supalla, T., \& Bavelier, D. (2015). The contribution of phonological knowledge, memory, and language background to reading comprehension in deaf populations. Frontiers in Psychology 6, doi: $\underline{10.3389 / \text { fpsyg.2015.01153 }}$

Hoeft. F., Meyler, A., Hernandez, A., Juel, C., Taylor-Hill, H., Martindale, J.L., et al (2007). Functional and morphometric brain dissociation between dyslexia and reading ability. Proceedings of the National Academy of Sciences, 104, 4234-4239.

Indefry, P. \& Levelt, W.J.M. (2004). The spatial and temporal signature of word production components. Cogn, 92, 101-144.

Kaufman, A.S., \& Kaufman, N. L. (2004). Kaufman brief intelligence test (2 ${ }^{\text {nd }}$ Edition). Bloomington, MN: Pearson. 
Koo, D., Kelly, L, LaSasso, C., \& Eden, G. (2008). Phonological awareness and short-term memory in hearing and deaf individuals of different communication backgrounds. Learning, Skill, Acquisition, Reading and Dyslexia, 1145, 83-99.

Lambon Ralph, M.A., Sage, K., Jones, R.W. \& Mayberry, E.J. (2010). Coherent concepts are computed in the anterior temporal lobes. Proc Natl Sci U S A, 107, 2717-22.

Landi, N., Frost, S.J., Mencl, W.E., Sandak, R., Pugh, K.R. (2013). Neurobiological bases of reading comprehension: Insights from neuroimaging studies of word level and text level processing in skilled and impaired readers. Read Writ Q, 29(2), 145-167.

Li, Y. Peng, D., Lui, L., Booth, J.R. \& Ding, G. (2014). Brain activation during phonological and semantic processing of Chinese characters in deaf signers. Frontiers in Human Neuroscience, 8, 211.

Lieberman, MD \& Cunningham WA (2009). Type I and Type II error concerns in fMRI research: re-balancing the scale. Social Cognitive \& Affective Neuroscience, 4, 423428. doi:10.1093/scan/nsp052

MacSweeney, M., Brammer, M.J., Waters, D., \& Goswami, U. (2009). Enhanced activation of the left inferior frontal gyrus in deaf and dyslexic adults during rhyming. Brain, 132, 1928-40.

Marschark, M., Sarchet, T., Convertino, C., Borgna, G., Morrison, C., \& Remelt, S. (2012). Print exposure, reading habits, and reading achievement among deaf and hearing college students. Journal of Deaf Studies and Deaf Education, 17(1), 61-74.

Mayberry, R.I., Chen, J-K., Witcher, P., \& Klein, D. (2011). Age of acquisition effects on the functional organization of language in the adult brain. Brain Lang, 119, 16-29. 
Mayberry, R.I., del Giudice, A.A., \& Lieberman, A.M. (2010). Reading achievement in relation to phonological coding and awareness in deaf readers: A meta-analysis. J Deaf Stud Deaf Educ, 16, 164-188.

McCandliss, B.D., Cohen, L., \& Dehaene, S. (2003). The visual word form area: expertise for reading in the fusiform gyrus. Trends Cogn Sci, 7, 293-299.

Miller, G.A., \& Chapman, J. P. (2001). Misunderstanding analysis of covariance. Journal of Abnormal Psychology, 110(1), 40-48.

Miller, P. \& Clark, M.D. (2011). Phonological awareness is not necessary to become a skilled deaf reader.J Dev Phys Disabil, 23, 459-76.

Miller, P. (1997). The effect of communication mode on the development of phonemic awareness in prelingually deaf students. J Speech Lang Hear R, 40, 1151-1163.

Neville, H. J., Bavelier, D., Corina, D., Rauschecker, J., Karni, A., Lalwani, A., et al. (1998). Cerebral organization for language in deaf and hearing subjects: biological constraints and effects of experience. Proc Natl Acad Sci U S A, 95, 922-929.

Patterson, K., Nestor, P.J., \& Rogers, T.T. (2007). Where do you know what you know? The representation of semantic knowledge in the human brain. Nat Rev Neurosci, 8, 976987.

Paul, P.V., Wang, Y., Trezek, B.J., \& Luckner, J.L. (2009). Phonology is necessary, but not sufficient: A rejoinder. Am Ann Deaf, 154, 346-356.

Price, C.J., Wise, R.J.S., \& Frackowiak, R.S.J. (1996). Demonstrating the implicit processing of visually presented words and pseudowords. Cereb Cortex, 6, 62-70. 
Pugh, K.R., Mencl, W.E., Jenner, A.R., Katz, L., Frost, S.J., Shaywitz, S.E., et al. (2000). Functional neuroimaging studies of reading and reading disability (developmental dyslexia). Ment Retard Dev Disabil Res Rev, 6, 207-213.

Purcell, J.J., Shea, J. and Rapp, B. (2014). Beyond the visual word form area: The orthography - semantics interface in spelling and reading. Cogn Neuropsychol, 31, 482-510.

Qi, S. \& Mitchell, R.E. (2012). Large-scale academic achievement testing of deaf and hard-ofhearing students: Past, present, and future. J Deaf Stud Deaf Educ, 17, 1-18.

R Core Team (2011). R: A language and environment for statistical computing. $R$ Foundation for Statistical Computing, Vienna, Austria. URL http://www.Rproject.org/

Schneider, J.W. (2013). Principles of Assessment of Aptitude and Achievement. In Saklofske, D.H., Reynolds, C.R., \& Schwean, V.L.. (Eds), The Oxford Handbook of Child Psychological Assessment (286-326). New York, NY: Oxford University Press.

Shipley, W.C. (1940). A self-administering scale for measuring intellectual impairment and deterioration. J Psych, 9, 371-377.

Stanovich, K. E. (1986). Matthew effects in reading: Some consequences of individual differences in the acquisition of literacy. Reading Research Quarterly, 21, 360-407. Stanovich, K.E. \& West, R.F. (1989). Exposure to print and orthographic processing. Read Res Quart, 24, 402-433. 
Sterne, A, \& Goswami, U. (2000). Phonological awareness of syllables, rhymes, and phonemes in deaf children. Journal of Child Psychology and Psychiatry, 41(5), 609625.

Supalla, T., Hauser, P.C., \& Bavelier, D. (2014). Reproducing American Sign Language sentences: cognitive scaffolding in working memory. Front Psychol, 5, doi: 10.3389/fpsyg.2014.00859.

Talairach, J. \& Tournoux, P. (1988). Co-planar stereotaxic atlas of the human brain. Thieme Medical Publishers: New York.

Wang, X., Caramazza, A., Peelen, M.V., Han, Z., \& Bi, Y. (2014). Reading without speech sounds: VWFA and its connectivity in the congenitally deaf. Cereb Cortex, first published online March 18, 2014 doi:10.1093/cercor/bhu044.

Waters, D., Campbell, R., Capek, C.M., Woll, B., David, A.S., McGuire, P.K., et al. (2007). Fingerspelling, signed language, text and picture processing in deaf native signers: The role of the mid-fusiform gyrus. NeuroImage, 35, 1287-1302.

Welcome, S., \& Joanisse, M. F. (2012). Individual differences n skilled adult readers reveal dissociable patterns of neural activity associated with component processes of reading. Brain and Language, 120, 360-371.

Wong, C., \& Gallate, J. (2012). The function of the anterior temporal lobe: A review of the evidence. Brain Research, 1449, 94-116. 


\section{Figure Captions}

Figure 1. The subgroup analysis (the top 10 and bottom 10 deaf readers) for the semantic task (vs. the control task) showed greater activity for the better deaf readers in the left inferior frontal gyrus (peak Talairach coordinates: $-57,+10,+17$ ), and the left middle temporal gyrus (peak Talairach coordinates: $-47,-43,+3$ ). No regions were more active for the least skilled readers. The statistical map displays the contrast up to $25 \mathrm{~mm}$ beneath the cortical surface on a template brain.

Figure 2. Significant voxel-wise partial correlations between brain activation (experimental task vs. control task) and behavioral scores. (A) During semantic processing, a region in the left anterior temporal lobe (peak Talairach coordinates: $-47,-5,-29$ ) showed a positive correlation between BOLD response strength and task accuracy. Two brain regions showed a positive correlation between BOLD response strength and reading ability: the left inferior temporal gyrus (peak Talairach coordinates: $-54,-29,-21$ ) and right inferior frontal gyrus (peak Talairach coordinates: $+47,+34,+3$ ). (B) During phonological processing, the left inferior frontal gyrus showed a positive correlation between BOLD response and task accuracy in two separate regions (peak Talairach coordinates: $-58,+10$, +17 and $-33,+24,+21$ ). Due to the positions of the clusters within the IFG and projection through the cortex, they appear as one merged cluster in the image. Statistical maps display group-wise correlations up to $25 \mathrm{~mm}$ beneath the cortical surface on a template brain. See the Results section for the range of correlation values across all voxels within each region. 


\section{Semantic Task: Skilled > Less Skilled}

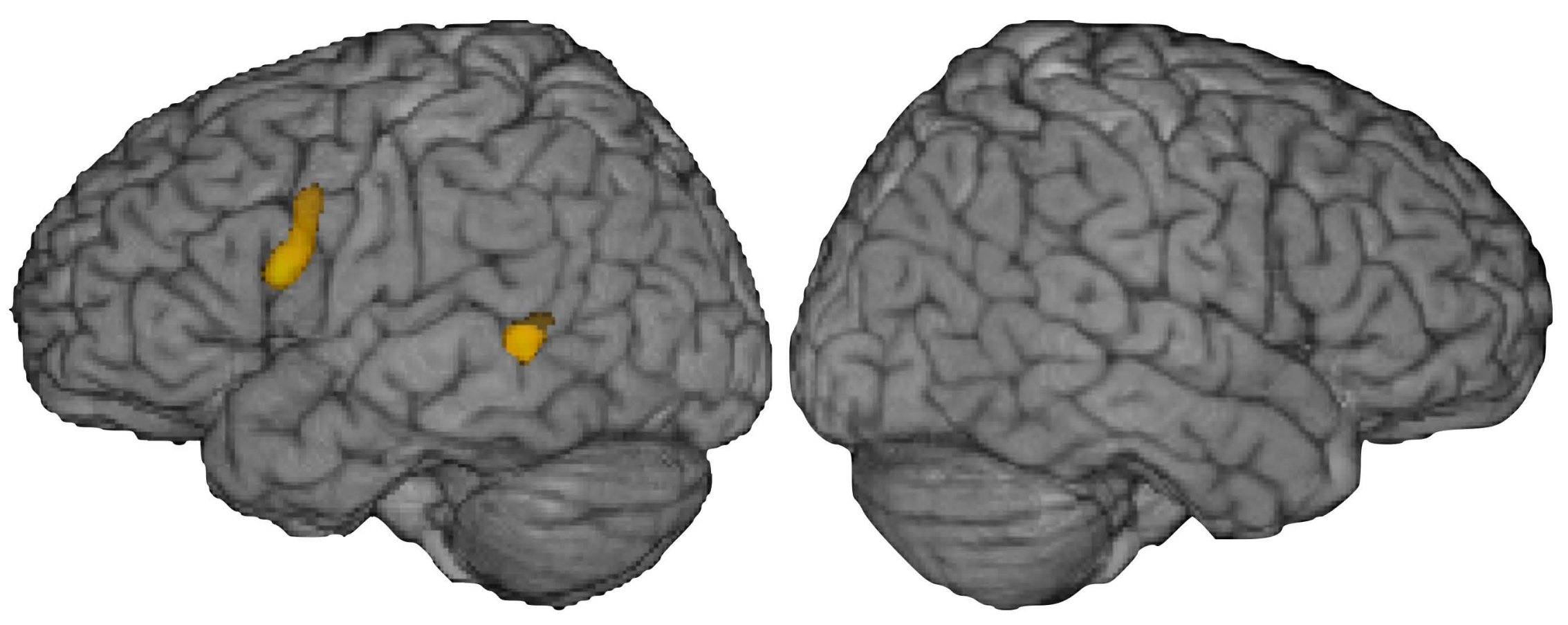

2.9

$t$-value

6.0 


\section{Partial Correlation Results}

A. Semantic Processing

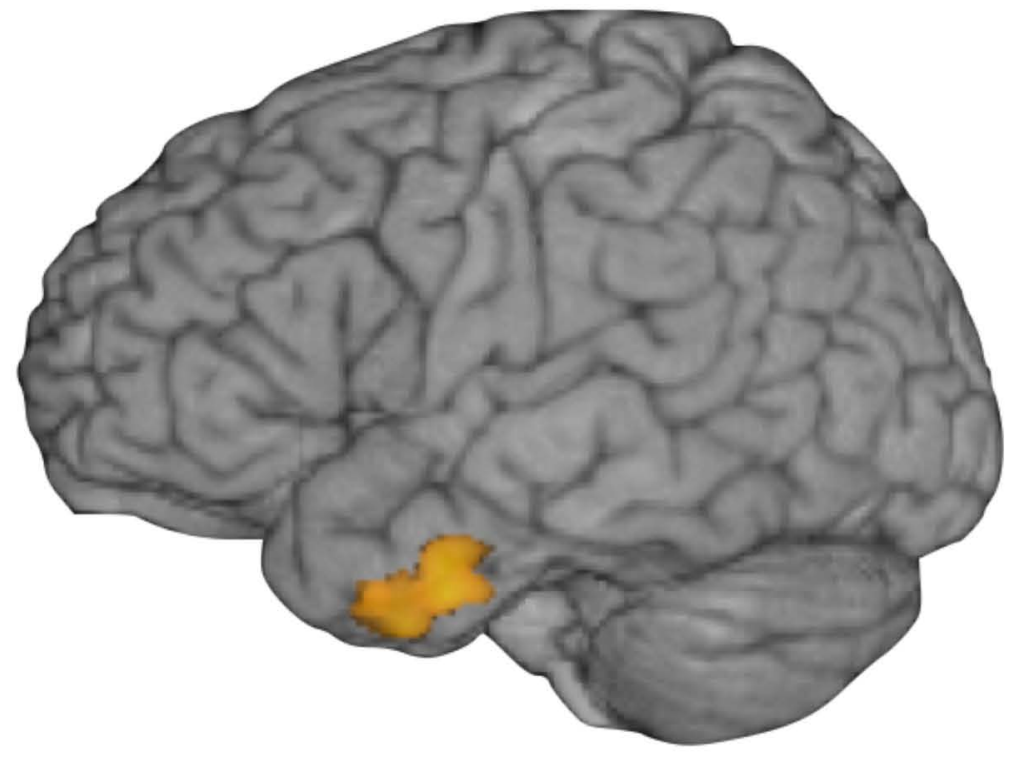

Task accuracy
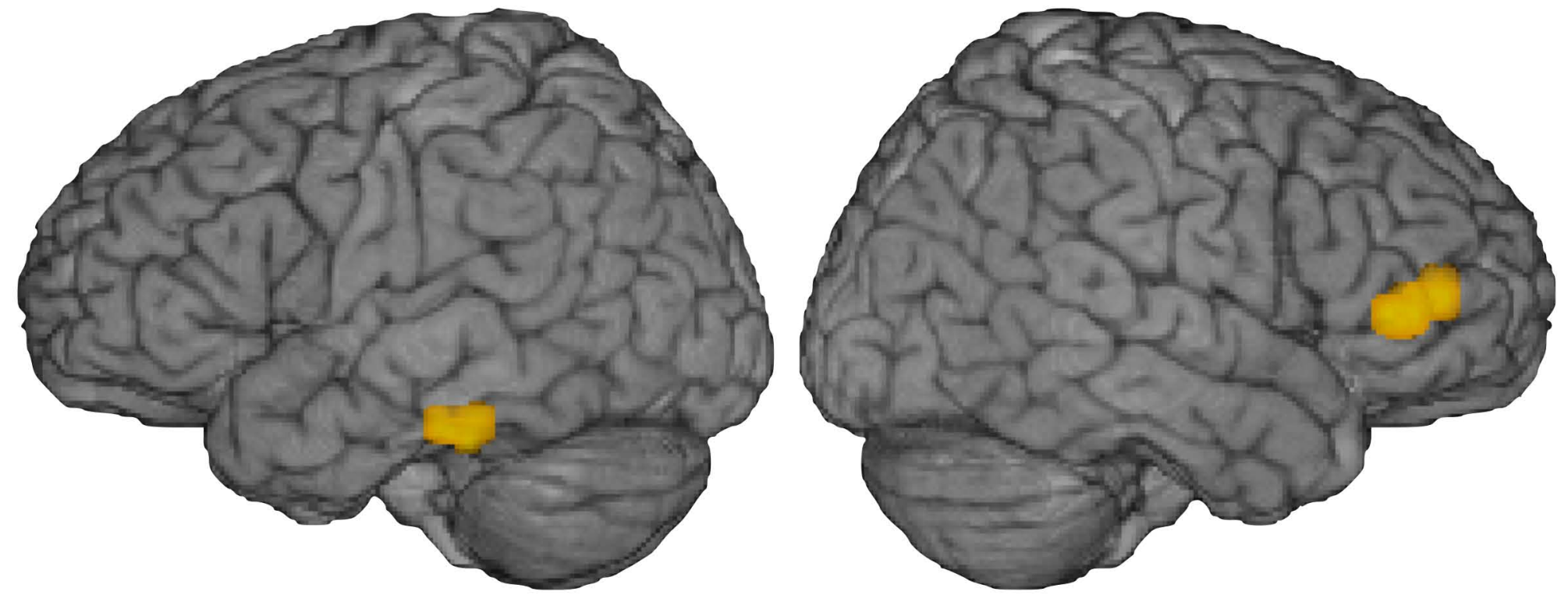

Reading ability

B. Phonological Processing

3.0

t-value

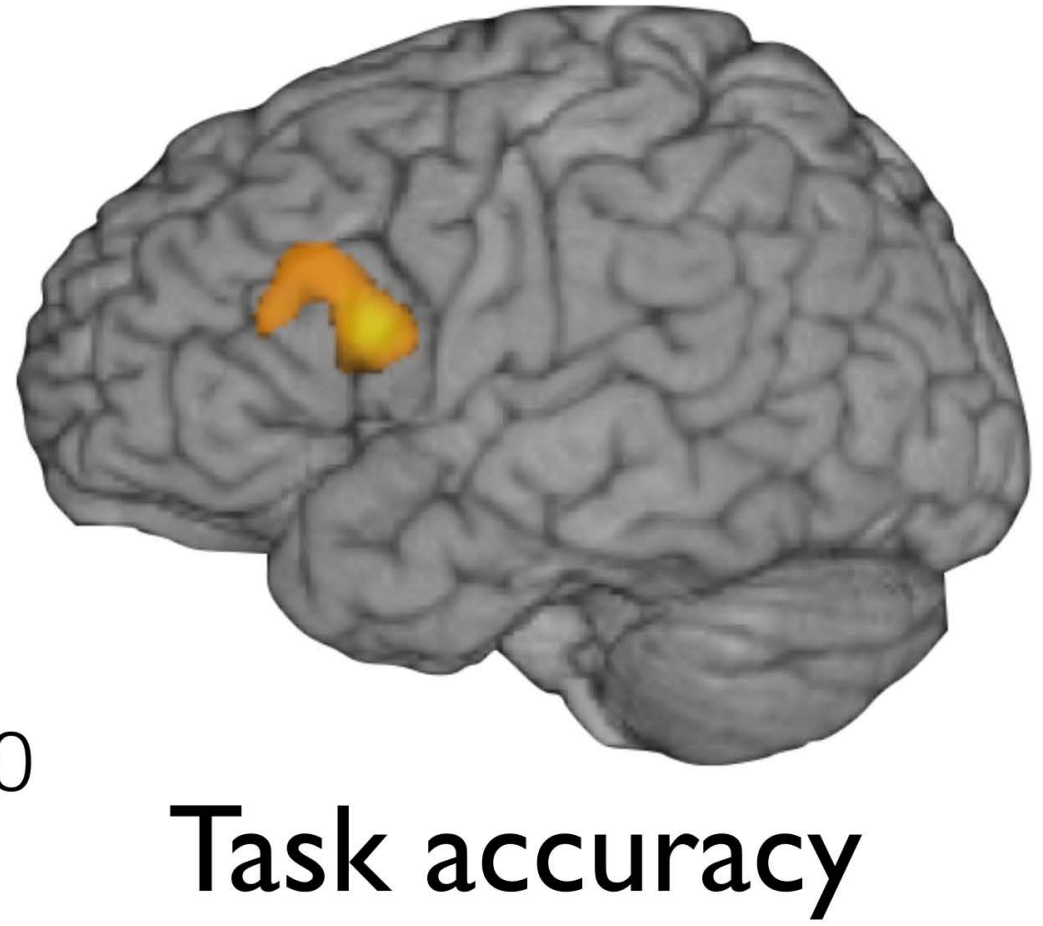


Table 1. Descriptive statistics and means for participant characteristics. Numbers in parentheses indicate standard deviations.

\begin{tabular}{|c|c|c|c|c|}
\hline & $\begin{array}{c}\text { Skilled } \\
\text { Readers }\end{array}$ & $\begin{array}{c}\text { Less Skilled } \\
\text { Readers }\end{array}$ & $\begin{array}{c}10 \text { Most Skilled } \\
\text { Readers }\end{array}$ & $\begin{array}{c}10 \text { Least Skilled } \\
\text { Readers }\end{array}$ \\
\hline Age & $28.07(5.2)$ & $27.57(3.6)$ & $28.9(5.2)$ & $27.5(4.2)$ \\
\hline Gender & 8 female & 8 female & 6 female & 6 female \\
\hline PIAT raw score ${ }^{\star \star \star}$ & $93.50(4.0)$ & $75.07(7.3)$ & $95.50(2.6)$ & $71.9(4.7)$ \\
\hline Range & $87-99$ & $64-86$ & $91-99$ & $64-80$ \\
\hline Mean reading age & 19.5 years & 12 years & 22 years & 11.5 years \\
\hline $\mathrm{KBIT}^{*}$ & $113.57(11.4)$ & $105.00(8.3)$ & $115.9(11.6)$ & $104.9(7.7)$ \\
\hline Shipley Vocabulary Test $(\max =40)$ * & $31.21(4.8)$ & $26.50(4.9)$ & $32.9(4.3)$ & $25.50(4.9)$ \\
\hline Author Recognition Test (raw score) $* * *$ & $14.21(7.4)$ & $5.43(3.9)$ & $14.21(7.4)$ & $5.43(3.9)$ \\
\hline Magazine Recognition Test (raw score) ** & $20.36(6.6)$ & $12.93(5.9)$ & $21.3(7.1)$ & $11.4(5.9)$ \\
\hline Phonological Awareness Test (\% correct) & $52.10(23.3)$ & $45.00(23.0)$ & $48.2(20.7)$ & $46.3(25.7)$ \\
\hline ASL-SRT $(\max =35)$ & $23.57(5.2)$ & $20.43(4.8)$ & $22.7(5.0)$ & $19.2(4.9)$ \\
\hline ASL experience & 10 native & 11 native & 8 native & 7 native \\
\hline \multicolumn{5}{|l|}{ In-scanner performance: } \\
\hline Phonological Task (\% correct) ${ }^{* *}$ & $76.30(15.3)$ & $61.88(8.9)$ & $80.63(15.0)$ & $62.0(7.8)$ \\
\hline Phonological Task (RT ms) & $1324(285)$ & $1172(208)$ & $1307(284)$ & $1150(193)$ \\
\hline Semantic Task (\% correct) ${ }^{\star \star *}$ & $94.20(2.8)$ & $88.9(3.8)$ & $95.0(1.7)$ & $87.8(3.7)$ \\
\hline Semantic Task (RT ms) & $1078(251)$ & $1016(134)$ & $1064(287)$ & $993(113)$ \\
\hline
\end{tabular}

PIAT = Peabody Individual Achievement Test; KBIT = Kaufman Brief Intelligence Test-second edition. ASL-SRT = American Sign Language Sentence Repetition Test.

Significant difference between groups: ${ }^{* *}=p<.001,{ }^{* *}=p<.01,{ }^{*}=p<.05$. 
Table 2. Partial correlation coefficients ( $p r)$ controlling for NVIQ between the reading measures, phonological awareness, and in-scanner performance.

$\begin{array}{llllll}1 & 2 & 3 & 4 & 5 & 6\end{array}$

\begin{tabular}{lllllllll}
\hline 1. PIAT & & & & & & \\
2. Author Recognition Test & $.68^{* * *}$ & & & & \\
3. Magazine Recognition Test & $.57 * * *$ & $.71 * * *$ & & & \\
4. Shipley Vocabulary Test & $.52^{* * *}$ & $.43^{*}$ & $.61 * * *$ & & & \\
5. Phonological Awareness Test & .14 & .16 & .23 & -.23 & & \\
6. Phonological decision in-scanner score & $.55^{* * *}$ & .32 & $.39 *$ & $.53^{* * *}$ & $.44 *$ & \\
7. Semantic decision in-scanner score & $.75^{* * *}$ & $.57 * * *$ & $.45^{*}$ & $.47 * *$ & .02 & $.40^{*}$ \\
\hline
\end{tabular}

$* * * \mathrm{p}<.005, * * \mathrm{p}<.01, * \mathrm{p}<.05$ 
Table 3. Brodmann areas (BA), peak Talairach coordinates, cluster sizes, and peak $t$-values for regions that were more active for the semantic task than the false font baseline task $(q \leq .05)$. See Emmorey et al. (2013) for sub-maxima within each cluster for the skilled deaf readers.

\begin{tabular}{lllllll}
\hline Region & BA & X & Y & $Z$ & $\begin{array}{l}\text { Volume } \\
\left(\mathrm{mm}^{3}\right)\end{array}$ & $t$-value \\
\hline
\end{tabular}

\section{Skilled Readers}

Frontal cortex

L superior frontal gyrus

$\begin{array}{cccccc}6 / 8 & -2 & +20 & +52 & 8661 & 6.78 \\ 6 & +5 & +20 & +45 & 1801 & 5.19 \\ 44 / 45 & -47 & +10 & +31 & 33057 & 11.40\end{array}$

R superior frontal gyrus

L inferior frontal gyrus

$44 / 45 \quad-47 \quad+10 \quad+31 \quad 33057$

11.40

Temporal cortex

L temporal pole

$\begin{array}{llllll}38 & -26 & +20 & -29 & 515 & 4.14\end{array}$

L middle temporal gyrus

$21 / 37$

$\begin{array}{llll}-58 & -50 & -4 & 21609\end{array}$

Cingulate cortex

L middle cingulate gyrus

$24+2 \quad+1 \quad+28 \quad 943 \quad 6.24$

\section{Less-Skilled Readers}

Frontal cortex

$\begin{array}{lcccccc}\text { L/R superior frontal gyrus } & 6 & +2 & +20 & +49 & 3130 & 4.91 \\ \text { L inferior frontal gyrus } & 47 & -33 & +34 & +3 & 728 & 5.63 \\ & 45 & -47 & +24 & +24 & 3044 & 5.34 \\ \text { R inferior frontal gyrus } & 45 & +54 & +20 & +28 & 943 & 4.08 \\ \text { L insula } & & -30 & +17 & +7 & 557 & 4.48 \\ \text { R insula } & & +30 & +20 & +7 & 943 & 4.69\end{array}$

Temporal cortex

L middle temporal gyrus

$\begin{array}{lll}-54 & -43 & -4\end{array}$

772 
Table 4. Brodmann areas (BA), peak Talairach coordinates, cluster sizes, and peak $t$-values for regions that were more active for the phonological task than the false font baseline task $(q \leq .05)$. See Emmorey et al. (2013) for sub-maxima within each cluster for the skilled deaf readers.

\begin{tabular}{|c|c|c|c|c|c|c|}
\hline Region & $\mathrm{BA}$ & $\mathrm{X}$ & $\mathrm{Y}$ & $\mathrm{Z}$ & $\begin{array}{l}\text { Volume } \\
\left(\mathrm{mm}^{3}\right)\end{array}$ & $t$-value \\
\hline \multicolumn{7}{|l|}{ Skilled Readers } \\
\hline \multicolumn{7}{|l|}{ Frontal cortex } \\
\hline $\mathrm{L}$ middle frontal gyrus & $9 / 46$ & -44 & +34 & +28 & 53465 & 9.35 \\
\hline $\mathrm{L}$ superior frontal gyrus & 6 & -2 & +17 & +45 & 11319 & 12.57 \\
\hline $\mathrm{R}$ superior frontal gyrus & $6 / 8$ & +5 & +20 & +45 & 7375 & 11.98 \\
\hline $\mathrm{R}$ precentral gyrus & 6 & +47 & +6 & +31 & 44076 & 7.64 \\
\hline \multicolumn{7}{|l|}{ Parietal cortex } \\
\hline L intraparietal sulcus & $7 / 40$ & -40 & -47 & +42 & 14320 & 11.47 \\
\hline $\mathrm{R}$ intraparietal sulcus & 40 & +30 & -57 & +38 & 14706 & 12.27 \\
\hline \multicolumn{7}{|l|}{ Temporal cortex } \\
\hline L fusiform gyrus & $20 / 36$ & -37 & -22 & -22 & 14449 & 5.12 \\
\hline \multicolumn{7}{|l|}{ Less-Skilled Readers } \\
\hline \multicolumn{7}{|l|}{ Frontal Cortex } \\
\hline $\mathrm{L} / \mathrm{R}$ superior frontal gyrus & 6 & +2 & +13 & +45 & 2486 & 5.92 \\
\hline \multirow[t]{2}{*}{$\mathrm{R}$ middle frontal gyrus } & 46 & +44 & +52 & +10 & 1157 & 5.87 \\
\hline & 8 & +44 & +24 & +35 & 1029 & 5.21 \\
\hline L middle frontal gyrus & 8 & -44 & +27 & +35 & 428 & 4.25 \\
\hline $\mathrm{R}$ inferior frontal gyrus & 47 & +26 & +41 & -4 & 557 & 4.62 \\
\hline L precentral gyrus & 6 & -47 & +3 & +35 & 472 & 4.89 \\
\hline L insula & & -30 & +7 & +10 & 1286 & 5.48 \\
\hline $\mathrm{R}$ insula & & +33 & +20 & +3 & 600 & 4.61 \\
\hline \multicolumn{7}{|l|}{ Parietal Cortex } \\
\hline $\mathrm{L}$ intraparietal sulcus & 40 & -40 & -43 & +42 & 2015 & 5.64 \\
\hline $\mathrm{R}$ intraparietal sulcus & 40 & +46 & -40 & +46 & 771 & 5.14 \\
\hline \multicolumn{7}{|l|}{ Temporal Cortex } \\
\hline L parahippocampal gyrus & 28 & -26 & -15 & -25 & 472 & 6.60 \\
\hline
\end{tabular}

